\title{
Use of virtual reality simulation in surgical training: a systematic review on predictive validity and current use in surgical curricula
}

\section{Aoife Feeley*, lain Feeley, Kalid Merghani and Eoin Sheehan}

Department of Surgery, Midlands Regional Hospital Tullamore, Co. Offaly, Ireland

${ }^{*}$ Corresponding author at: Department of Surgery, Midlands Regional Hospital Tullamore, Co. Offaly, Ireland.

Email: aoifefy@gmail.com

Date accepted for publication: 27 January 2021

\section{Abstract}

Background: Simulated surgical learning is an evolving training modality for surgical trainees. Its use in transferring simulation-based skills to the operating room is an integral aspect of its use as a pedagogical tool for surgical trainees in an era of reduced working hours and fewer intra-operative opportunities. These systems may allow trainees to upskill in simulated scenarios leading to improved skillsets and patient safety. The aim of this review was to evaluate if acquisition of surgical skills developed in simulated procedures results in improved intra-operative performance and whether this can be integrated into current surgical curricula. Methods: A systematic search was conducted using PubMed, OVID Medline and CINAHL. Articles included were based on specific inclusion and exclusion criteria. Critical appraisal tools were used to assess each article's authenticity, applicability and quality of results. Results: Twenty-six studies were reviewed in full and included in this review according to PRISMA guidelines. Thematic analysis yielded four main themes: predictive validity, surgical curriculum, timing of training, clinical outcomes. All studies demonstrated validity. Conclusion: A heterogeneous group of studies demonstrated mixed findings in the predictive validity of virtual reality learning. However, adaptation into surgical curricula in conjunction with other forms of surgical education yielded positive results, with predictive validity demonstrated in surgical trainees. Further research is required to elicit optimal training stages and use of simulation in development of non-technical skills.

Keywords: simulation; simulation training; surgical trainees; predictive validity

\section{Introduction}

Augmented and virtual reality (VR) software is a rapidly evolving and expanding modality of training within surgery, enabling trainers and surgical residents to assess cognitive functions, including problem solving and object recognition, ${ }^{1}$ and providing an initial experience of the movements required to perform complex tasks. ${ }^{2}$ In addition, rapid technological and medical advancements behove surgical training bodies to maintain continuing professional development and to fortify the practice of testing newly developed surgical techniques and devices. Incorporation of virtual intelligence systems into health care systems requires careful consideration of several factors, including patient safety, cost effectiveness and suitability for provision of training. ${ }^{3}$

Within current surgical specialty training, care is taken to ensure trainees are guided to an appropriate level at each stage according to their experience and technical skillset, ${ }^{4}$ the end goal being to maximize surgical skillsets in surgical residents while minimizing the potential of adverse outcomes to patients. Integration of VR could augment current competency-based training systems evolving in health care systems. When first introduced, laparoscopic procedures were associated with higher rates of complications and longer operative times, mitigated by the introduction of dedicated training on the method. ${ }^{5}$ With dedicated laparoscopic simulation training, learning curves conventionally seen in training could occur in safe environments with no associated risk to patients. In this regard, augmented reality in surgical simulation has been proffered as a potential solution to learning curves ${ }^{6}$ associated with new technologies and techniques, ultimately leading to improved patient safety in the real world of surgical practice.

Recent global issues have highlighted the potential role of VR in helping develop and maintain surgical skills both in 
surgical trainees and fully qualified surgeons. With the onslaught of COVID-19 affecting hospitals worldwide, and an increase in hospital resources directed towards combatting the effects of the pandemic, elective lists were cancelled and surgical trainees faced loss of surgical skills. With surge plans $^{7,8}$ in use in many institutions, time away from the hospital required novel techniques to prevent loss of skill from diminished caseloads. ${ }^{9}$

With recent advancements in augmented and VR simulation, a plethora of data now exists examining the potential use of simulation training in surgical specialties, including ophthalmology, ${ }^{10}$ gynaecology, ${ }^{11}$ neurosurgery ${ }^{12}$ and orthopaedics. ${ }^{13}$ Although research has demonstrated a benefit in both laparoscopic and endoscopic training, ${ }^{5,14}$ simulation training has yet to be proven for broader surgical specialties, because proficiency in surgical procedures cannot be assessed based on simulator metrics alone. ${ }^{15}$ It is important to consider if tangible evidence exists to show that simulation training confers intra-operative skills and benefits clinical outcomes. ${ }^{16,17}$

\section{Objective}

This review aims to systematically evaluate the literature published on the use of VR simulation and surgical applications to assess (1) the predictive value of VR training and (2) the feasibility of VR in surgical training curricula.

\section{Methods}

A systematic review of PubMed, Ovid Medline and CINAHL, was carried out. Terms used in the search included a combination of "virtual reality", "simulation training", "surgical training", "surgical performance", "predictive validity", "warm-up training" using Boolean characters "AND", "OR". Study selection was carried out between July 2020 and September 2020. Filters applied to the database searches included English language. Publication date parameters were set from 2009 to September 2020.

Titles and abstracts of each study were read to identify relevant studies. If the inclusivity of the study was uncertain, the study was read in full. Inclusion and exclusion criteria were applied to relevant studies identified, and reference lists of relevant articles were evaluated for studies suitable for inclusion (Fig. 1). Data extraction was carried out independently by reviewers. The results were collated and presented in tabulated form. Meta-analysis was not possible due to the heterogeneity of the findings. However, common themes across studies included in this review were identified and evaluated.

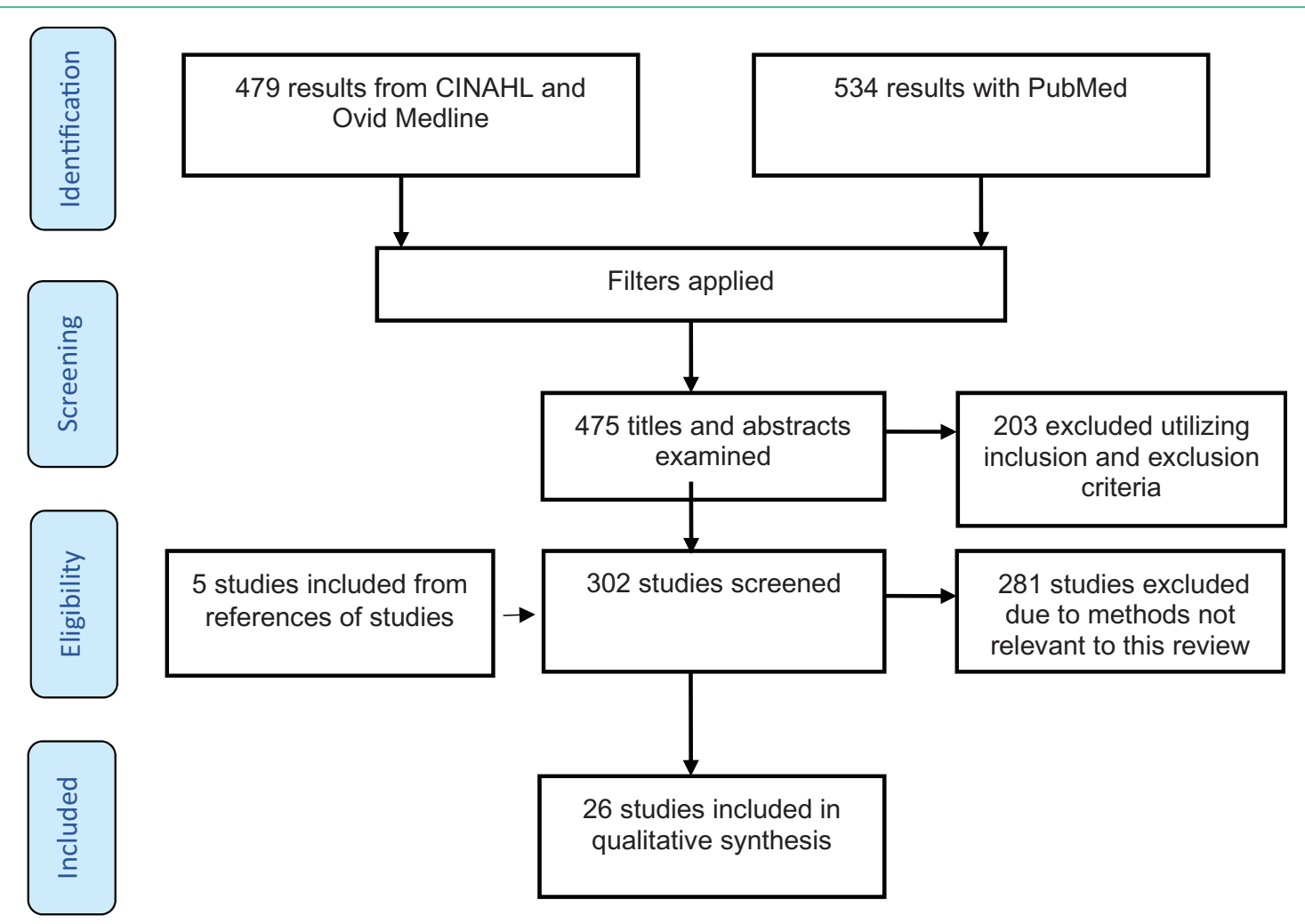

Figure 1. Flowchart for selection of studies included for review. 
Study quality was assessed using quality assessment tools according to the Cochrane guidelines; risk of bias tools, Rob2 (Fig. 2) ${ }^{18}$ and Robins-I (Fig. 3), ${ }^{19}$ were used to evaluate the validity of the studies evaluated in this review.

\section{Inclusion criteria}

- Studies using biological tissue in the final assessment

- Studies adapting VR into surgical curricula with clinical outcomes assessed

\section{Exclusion criteria}

- Studies focused primarily on the use of VR in pre-operative planning

- Studies assessing face and construct validity of their VR simulators

- Systematic reviews

- Studies analysing VR in gastrointestinal endoscopy
- Studies using simulation-based assessment only

\section{Results}

\section{Search strategy}

The search criteria returned 1003 articles. After complete evaluation, 26 articles were included for review, five of which were from manual searches (Fig. 3). Articles returned were grouped thematically into studies evaluating primarily predictive validity (Table 1) and studies evaluating use of VR in surgical curricula (Table 2).

\section{Methodologies}

One study ${ }^{20}$ used an interventional method comparing students and trainees in performance after simulation exposure. One study ${ }^{36}$ used a retrospective observational design to assess learning curves obtained with VR compared with other learning modalities. Two studies ${ }^{21,25}$ used a before and after design to assess the use of VR in improving surgical performance. Two studies used a randomized controlled trial with no blinding. Ahlborg et al. $^{24}$ failed to achieve

Bias arising from the randomization process

Bias due to deviations from intended interventions

Bias due to missing outcome data

Bias in measurement of the outcome

Bias in selection of the reported result

Overall risk of bias

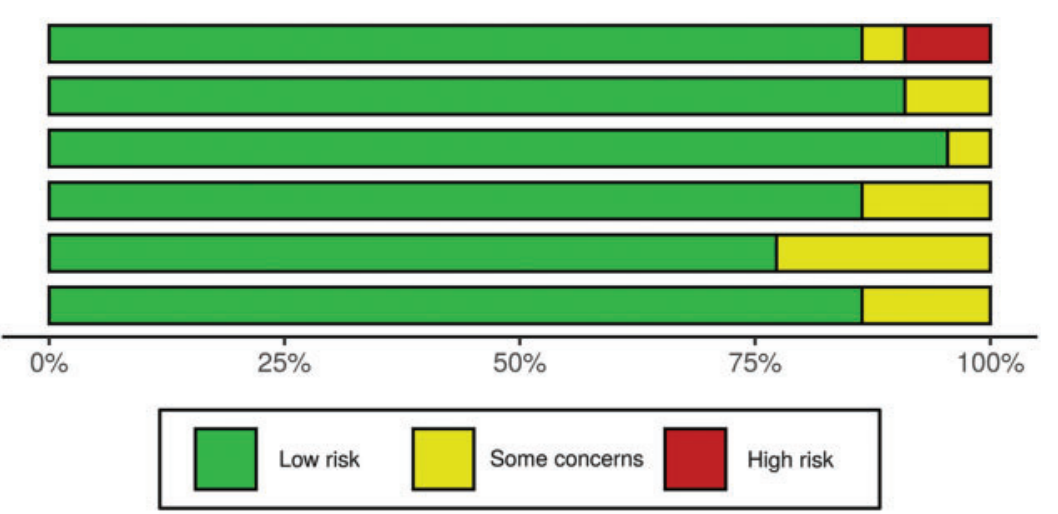

Figure 2. Rob2 risk of bias tool demonstrating the validity of the RCTs included.

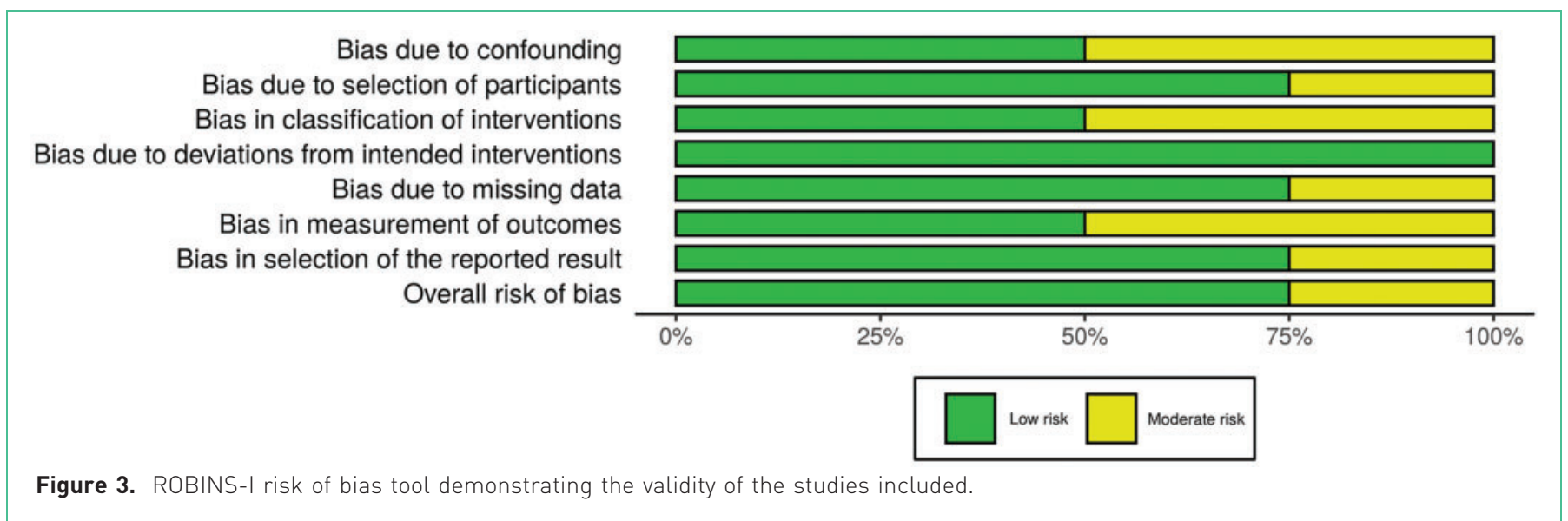




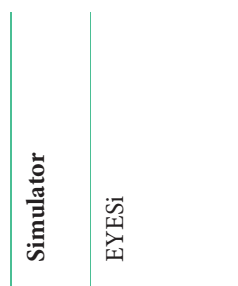

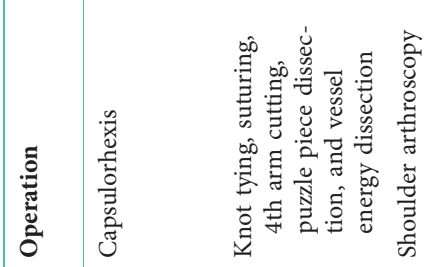

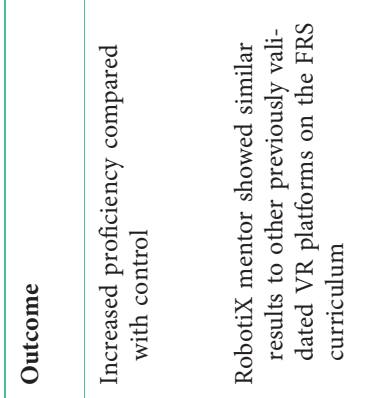

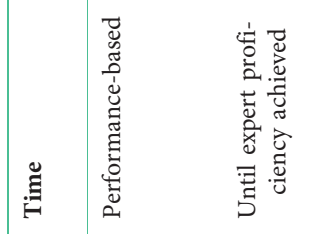

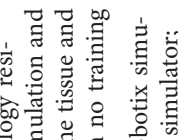

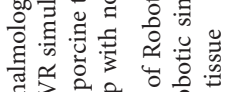

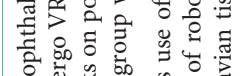

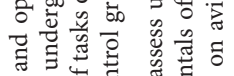

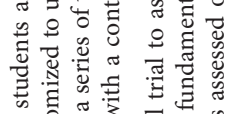

$\stackrel{\frac{\pi}{7}}{\frac{\pi}{\sigma}}$

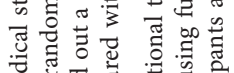

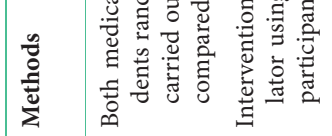
(6)

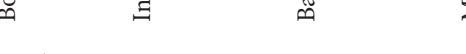

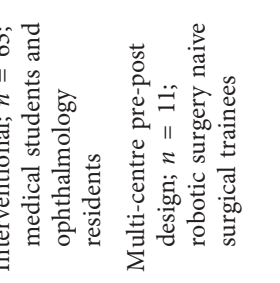

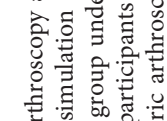

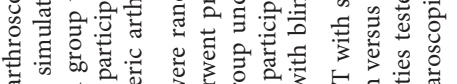

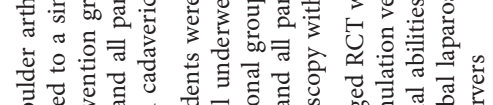

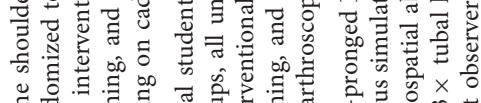

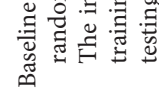

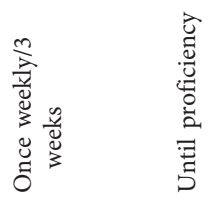

远

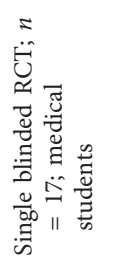

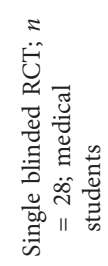

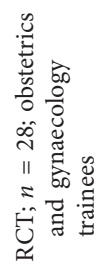

离

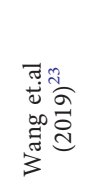

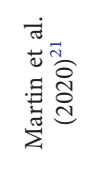

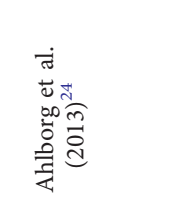

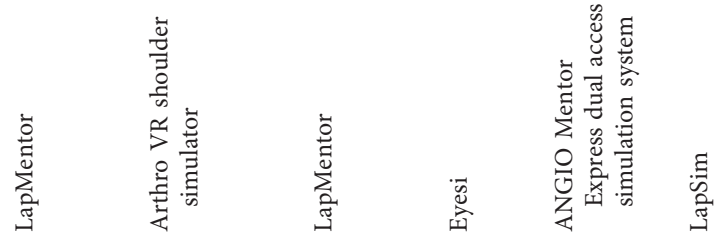

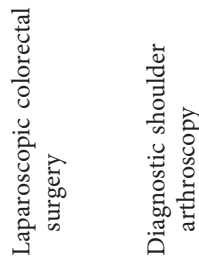

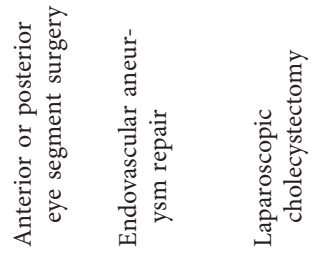

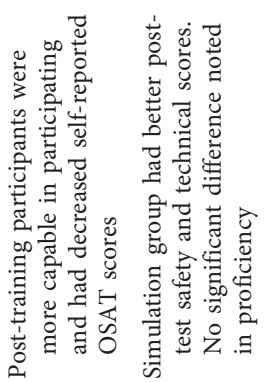

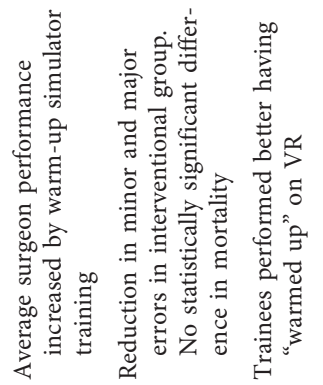

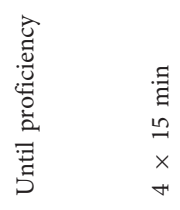

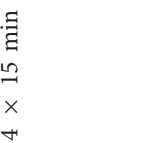

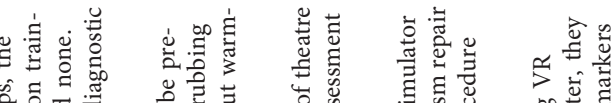
$\begin{array}{llll} & \\ 0 & 0 \\ 0\end{array}$

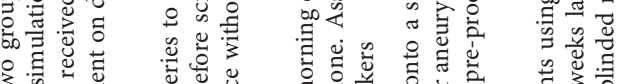

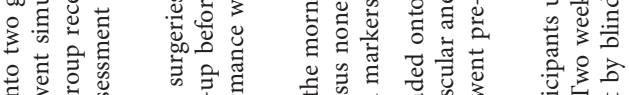

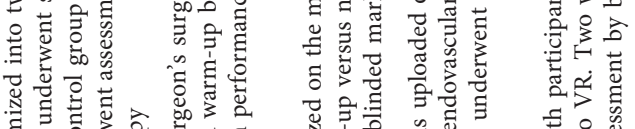

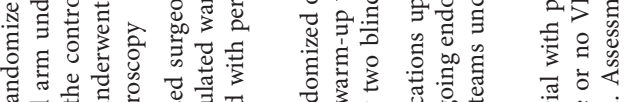

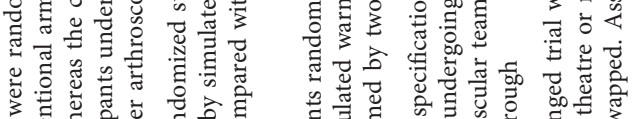

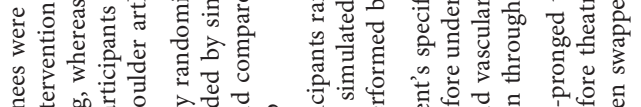

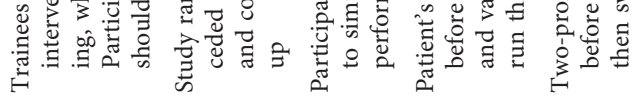

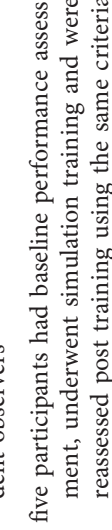

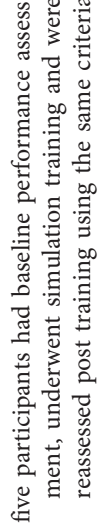

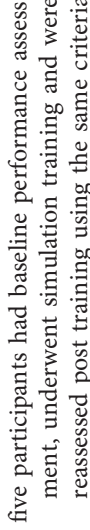

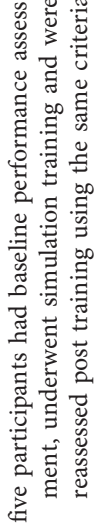

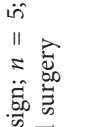

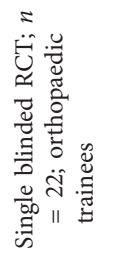

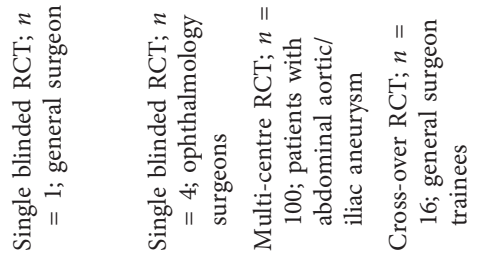

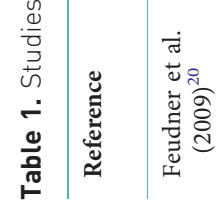

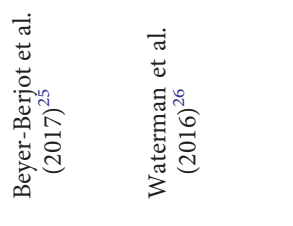

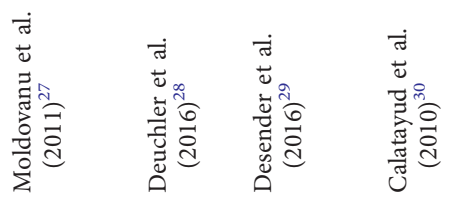


In

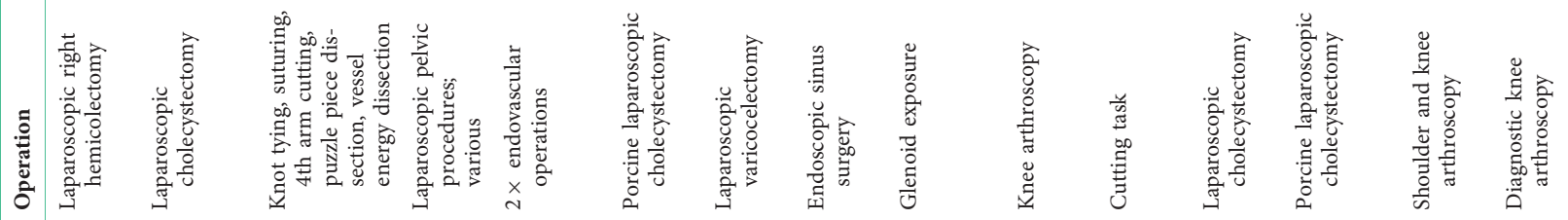
!UM II 111

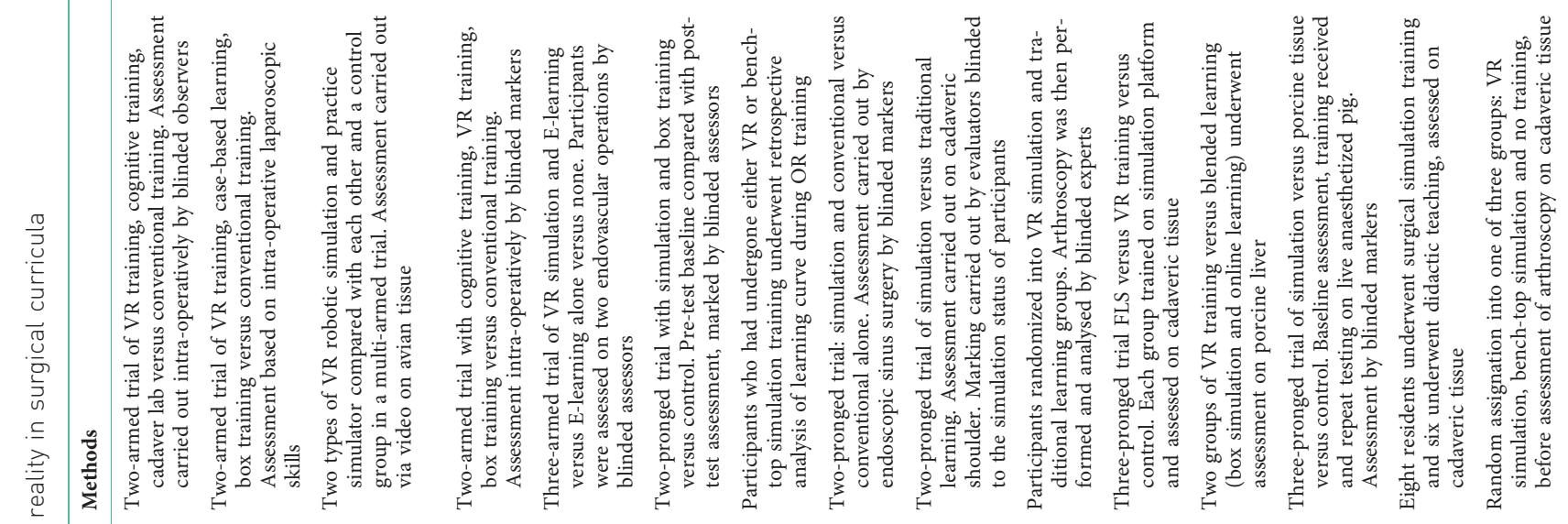

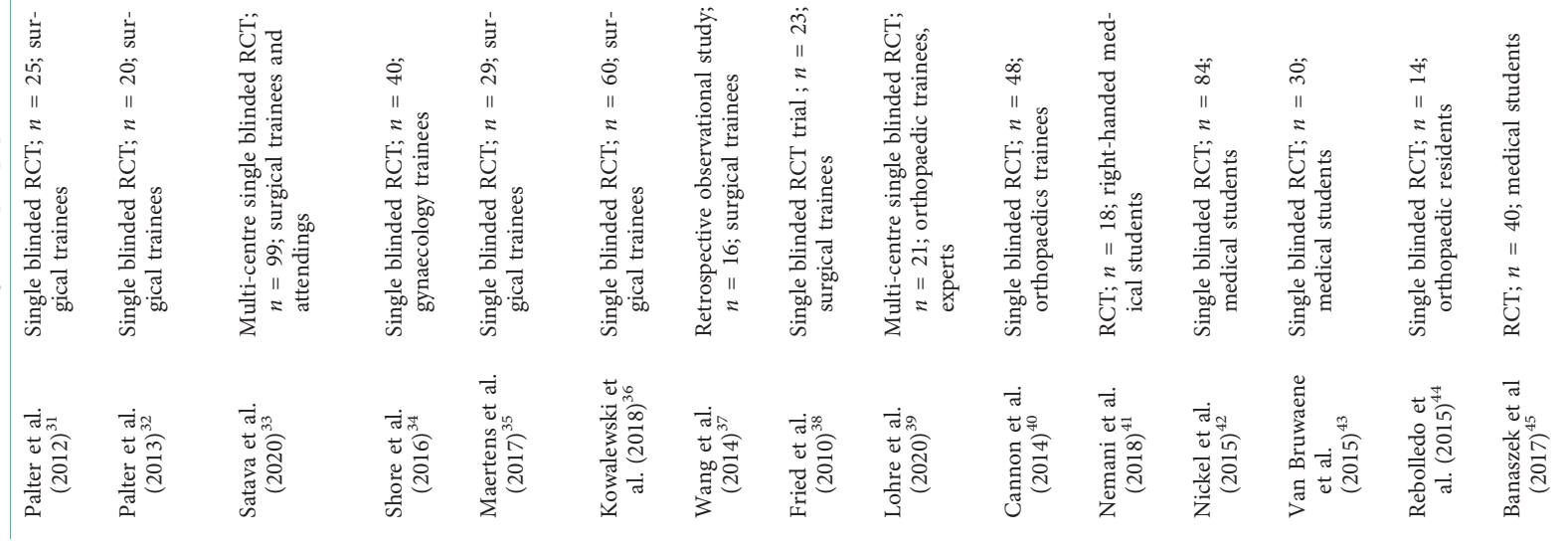


inter-rater reliability and Nemani et al. ${ }^{40}$ used no subjective assessments in the trial. Seventeen studies used a single blinded randomized controlled trial design.

\section{Use of models}

Feudner et al. ${ }^{20}$ used porcine wet labs. Two studies ${ }^{35,41}$ used porcine models with a pulsating organ perfusion (POP) trainer. Van Bruwaene et al. $^{42}$ used live anaesthetized pigs. Two studies $^{21,32}$ used avian tissue. Five studies ${ }^{22,38,40,43,44}$ used cadaveric models. Two studies ${ }^{26,39}$ used real patient arthroscopy as the final assessment for participants. Fried et $\mathrm{al}^{37}$ used endoscopic surgery in the final assessment. Three studies ${ }^{24,25,34}$ used real patient operations in the final assessment. Shore et al. ${ }^{33}$ used a variety of laparoscopic pelvic operations in the final assessment, each categorizes as mild, moderate or difficult by the blinded assessors based on the anatomy and co-morbidities. Four studies ${ }^{27-29,45}$ used real patients to measure the effects of pre-operative simulation.

\section{Time metrics}

Ten studies used a predetermined time for the VR simulation arm. ${ }^{22,23,26,30,31,35,40,41,43,44}$ Eleven studies used a proficiency-based model. ${ }^{20,21,24,25,32-34,37,39,42}$ One study did not specify the method used. ${ }^{38}$

\section{Operations}

Six studies ${ }^{22,23,26,39,43,44}$ used arthroscopic procedures in the final assessment. Twelve $e^{21,24,25,27,29-32,35,36,41,42}$ post-test procedures were laparoscopic, with two endovascular assessments, ${ }^{28,34}$ one endoscopic sinus procedure, ${ }^{37}$ and two procedures involving the eye. ${ }^{20,45}$ One procedure focused on exposing the glenoid. ${ }^{38}$

\section{Discussion}

\section{Predictive validity}

Training on simulator-based surgical cases has been demonstrated to improve subsequent scoring on assessment using these models, indicating that simulated training allows development and retention of skills in the simulated environment. ${ }^{46-48}$ Predictive validity of simulation-based training is an integral aspect of the benefits of simulated-based learning; ensuring the training time put into simulation-based modules will result in improved proficiency in the operating room is key to the training method being an effective alternative to real patients, cadaver models, and animal tissue. Assessment of proficiency in the studies included in this review focused on the transferability of skills obtained in the virtual world to the real one.
Studies on direct translatability of VR-obtained skills to real tissue were evaluated in this review. Given the heterogeneity of the subjects studied, including a number evaluating the efficacy of VR in medical students, the use of a variety of tissue is expected. Traditional learning methods for medical students and novice trainees include proxy tissue and cadavers, and in times of depleted available tissue, alternative home-made supplies have been used. ${ }^{49}$ With ongoing development in the technological apparatus used for learning new skills, it is reasonable to compare the new method of teaching with previously established methods of training. A potential advantage of simulation training is its ability to correctly identify the relative skill and experience level of the user, which may also be useful in delineating the relative safety of the surgical trainees. ${ }^{50,51}$

Four studies ${ }^{20,35,41,42}$ in this review looked at transfer of skills to animal tissue from VR alone, with mixed findings. Feudner et al. ${ }^{20}$ noted an increase in proficiency across several parameters using porcine substitutes, which would indicate the transfer of simulated skills to biological tissue. Van Bruwaene et al. ${ }^{42}$ used live anaesthetized pigs in the final assessment, with cadaver-trained individuals performing better than those trained using VR. These authors noted that the full translatability of the study could not be fully assessed due to the model of tissue used and its relative differences to human cases. The animal training group used porcine tissue in their training, which was the same as final tissue used, and thus may have had an impact on their final performance, a confounder that was not addressed in the limitations. Two studies evaluated the translatability of simulation-achieved skills using animal tissue on POP trainers. ${ }^{35,41}$ Nickel et al. ${ }^{41}$ found comparable proficiency between groups; VR resulted in faster times to completion, and controls demonstrated superior knowledge. Kowalewski et al. ${ }^{35}$ compared the use of both VR and box training with traditional surgical training, finding the intervention group were significantly faster, with more laparoscopic skill demonstrated compared with the controls. Despite this, the study noted there was no correlation between the post-test VR simulation outcome and the POP porcine laparoscopic cholecystectomy, except for time to completion. The methodology used in this study precluded full comparison of outcomes between simulated and porcine performance, and should be considered a confounder in the study's outcomes. Satava et al. ${ }^{32}$ compared two VR models and a robotic simulation with avian tissue used in the final assessment. No difference in proficiency was demonstrated for any of the models used or the control group. The authors noted the control group had a higher level of fully qualified surgeons compared with the interventional groups, reflected in the superior performance of the 
control group in the pre-test assessment. An additional limitation was the concern regarding the stability of the tissue, which could have affected the outcome of some of the findings. However, Martin et al., ${ }^{21}$ who followed on from Satava et $\mathrm{al}^{32}$ to ascertain the predictive validity of a VR robotic platform for avian tissue, raised no concerns regarding the validity of the tissue.

Six studies used cadaver models in the final assessment. Cadaveric tissue was used most often for medical students because it was the best training model given that it is the most realistic proxy to the operating room. Despite this, limitations include the limited number of available specimens and durability of tissue, including change in tissue tension and lack of biological feedback. Two studies found no significant difference in performance between simulated groups and the control; $;^{22,23}$ the other four demonstrated improved metrics for VR-trained participants. ${ }^{38,40,43,44}$

Nemani et al. ${ }^{40}$ compared the use of VR training with a Fundamentals of Laparoscopic Surgery (FLS) module against a previously established simulation curriculum in the final assessment using cadaver tissue. Although both the FLS and VR group outperformed the controls, the FLS group were most proficient based on the metrics measured. As no measurement of surgical skills required to complete the task was carried out, this must be a considered a limitation to the study. Ahlborg et al. ${ }^{24}$ failed to achieve interrater reliability and used operating time to demonstrate the beneficial effect of simulation training on surgical skills. The authors also recorded self-efficacy measurements of the participants, noting that the simulated cohort recorded higher levels, which have previously been correlated to faster operating times. There is consequently correlation bias as to whether faster operating times were secondary to higher self-efficacy scores or due to increased proficiency.

The predictive value of VR simulation has been previously evaluated in gastrointestinal endoscopy. ${ }^{52,53}$ Other endoscopic surgeries are becoming more common, creating their own challenges in mastery for surgeons of all skill levels. Endoscopic surgery has changed the face of surgical training due to the manner in which skills are obtained, and simulated environments are ideally placed for development of these skills. Interest in simulated laparoscopic, endoscopic and arthroscopic procedures has resulted in a recent focus in this area, with inconsistent findings. One study evaluated the predictive value of an endoscopic simulator to in vivo endoscopy. ${ }^{37}$ They found the simulation-trained residents were faster, made fewer errors, were more confident, and had higher levels of dexterity than controls. However, this is not ubiquitous across predictive validity studies. One study looking at the predictive validity of simulation training in real patient cases noted there was a significant difference in checklist skills $(P=0.031)$; however, no significant difference was found in visualization or time taken. ${ }^{39}$ No significant difference was noted in the proficiency levels between the two groups $(P=0.061)$, which the authors attributed to an outlier. Waterman et al. ${ }^{26}$ found similar results using real patient diagnostic arthroscopy to assess transferability; no significance was found between the groups. Wang et al. ${ }^{23}$ assessed the transferability of simulator-obtained arthroscopy skills on cadaveric specimens and noted that although the post-training simulation assessment demonstrated improvement in the simulator groups compared with the control group, this improvement was not replicated in the cadaveric assessment, echoing similar studies evaluating other methods of surgical teaching. ${ }^{54}$ Two studies in this review found simulation-trained participants performed better $(P<0.05)^{44}$ with more complete injury grading indexes ${ }^{43}$ than the controls in arthroscopic assessment. One study ${ }^{38}$ evaluated a simulation group against journaleducated controls in cadaveric shoulder joints, finding the simulation group had comparatively higher scores and were faster to completion. Only knowledge was comparable between the groups. The heterogeneity of the results perhaps helps delineate the relative limitations of VR training in its current form.

Studies on the direct translation of skills from the virtual model to real patients are the most beneficial because this removes potential confounders and allows analysis from direct comparisons. Twelve studies used real patients in the final assessment, four of which looked solely at VR to patient skill transferability. ${ }^{24-26,39}$

One study ${ }^{25}$ evaluated the use of simulation training in a group of colorectal trainees, with their pretraining test as controls. Although their level of participation increased intra-operatively $(0 \%-85 \%)$, rather surprisingly their selfreported overall satisfaction scores were lower for the post-training operations. This is potentially due to the increased participation reflecting that the trainees were the primary operator for the first time, which had a negative impact on their self-reported skills. Ahlborg et al. ${ }^{24}$ found the interventional group were faster to completion.

Shore et $\mathrm{al}^{33}$ noted an improvement in performance in a multimodal interventional group. Final assessment was carried out intra-operatively by blinded assessors.

The mode by which VR training would have an effect on intra-operative performance has not yet been established. Palter et al. $^{31}$ found a significant difference in the simulator-trained group in the final assessment. The intervention group underwent multiple methods of adjunctive learning, 
which may explain the stark contrast between the two groups in the final assessment. Unlike the intervention group, the control group underwent a significant learning curve between the first and second operations; the authors attributed this curve to having occurred on the simulator. This would signal that translatability was achieved by the participants in the intervention group. One study ${ }^{36}$ looked at the effect of VR and its effect on the intra-operative learning curve, using metrics including time, complications and recurrence to establish this. Having established the curve consisting of a learning phase, improvement phase and platform phase, the use of VR was found to change the curve compared with that of the control group of box-trained surgical trainees. VR shortened the mean operative time, primarily in the learning phase of the curve. The improving phase of the curve was shorter with fewer cases in the VR group. No conventional training group was used, which limits analysis. VR was demonstrated to have altered the curve and improved mean operative time with particular effect in the early stages of training. This would correlate with previously established data regarding the early ascension of the curve in the untrained..$^{55}$ Learning curves reflect the level of experience, ${ }^{56}$ and the ceiling effect previously noted in VR studies is demonstrated by the earlier rise to platform level in the VR group. This is potentially due to quick adaptation to the tasks on the simulator, which results in a slower progression and indicates there is scope for more difficult tasks. ${ }^{57}$

Four studies looked at the use of VR simulation in preoperative preparation and outcomes from this. One study ${ }^{28}$ used patient-based metrics to do a simulated "run through" using VR in a simulated environment. This resulted in fewer intra-operative minor and major errors. Three studies looked at the effect of VR directly before surgery, ${ }^{27,29,45}$ finding improved surgical performance compared with surgeries without VR simulation before scrubbing. This is likely due to a "warm-up" effect of a simulated run through of procedures rather than acquisition of technical surgical skills obtained through VR. However, it does highlight the potential for VR to aid in training in nontechnical skills central to surgical training and performance.

\section{Surgical curriculum}

Conventional methods of surgical training remain within the apprenticeship model. Curriculum models vary across health care services and within different surgical specialties, and novel programmes are implemented to optimize training. The introduction of virtual training into curricula would require feasibility, ease of access and use, and an objective benefit seen from the introduction of this method to the surgical trainees regarding skill, comprehensibility, and enjoyment. Surgical learning adjuncts used in curricula include bench-top models, boxtraining sets and cadaveric models. Of the studies assessing VR against current curriculum methods, two compared outcomes against didactic or E-learning programmes, ${ }^{34,43}$ five evaluated VR against other procedural adjuncts in use, ${ }^{36,40-}$ 42,44 and six looked at VR compared with conventional surgical models. ${ }^{30,31,33,37-39}$ Maertens et al. ${ }^{34}$ noted the superiority of simulation training and supplemental online learning over online modules alone, which was superior again to their current surgical training programme. The programme implemented took an average of 8 months to complete and required an average of 13 simulation sessions to achieve competency. Nemani et al. ${ }^{40}$ compared the use of VR against a previously validated learning adjunct in surgical training, FLS; simulation training was carried out on the FLS box trainer. FLS has previously been adopted into surgical curricula; its versatility has been found to be useful in progressing surgical trainees' skillsets. The study found that although both VR and FLS improved performance metrics compared with the control group, no significant difference was noted between the two interventional groups, indicating that VR is a viable alternative to this previously established learning adjunct. Palter et al. ${ }^{30,31}$ incorporated VR training into surgical curricula in conjunction with other surgical training adjuncts to compare it with current training practices. It was demonstrated comprehensively that participants in the interventional arm performed better; a stark difference was noted, particularly in the initial operations, ${ }^{31}$ a finding mirrored by Shore et al., ${ }^{33}$ who used four additional methods of training in the intervention arm. As the use of VR in these studies was carried out in the same intervention arm with other methods, it is difficult to attribute this finding to VR use, however it is possible that the learning curves that surgical trainees traditionally undergo in the early stages of their training were experienced on simulated models with subsequent superiority intra-operatively compared with those without simulation exposure. Participants in the control arm in all these studies underwent the standard curriculum of surgical trainees, and as a control, are optimal to delineate the additional benefits these methods can provide while being a feasible addition to the rigours of trainee curricula. One study evaluated simulation against conventional learning using both surgical trainees and experts in the specialty. ${ }^{38}$ The authors found that trainees found the simulation enjoyable and easy to use, and a benefit to continued use for themselves $(P=0.009)$ and for novices $(P=0.08)$.

Wang et al. ${ }^{36}$ noted the VR system was unsuitable for training ligation of the spermatic cord; ascertaining that it could not simulate the procedure and was therefore not applicable 
to all aspects of surgical training. This study was carried out in 2014, and the potential for expansion of VR simulation, based on available technology, should always be considered.

Desender et al. ${ }^{28}$ and Shore et al. ${ }^{33}$ used a fully immersive simulation in their studies, adding a potential confounder to the outcome as a sole measure of the effects of VR training. However, this method provides the addition of non-technical factors that are integral in the difference between novices and experts. ${ }^{15}$ Use of full teams in VR simulation creates a more realistic scenario in which to develop skills pertaining to the operating theatre, such as communication, situational awareness, planning, and teamwork and thus should be taken into consideration when planning VR in the context of complete surgical training. This concept has been outlined previously in the literature ${ }^{58}$ indicating communication and interpersonal skills should be a focus in education.

\section{Timing of training}

Surgical training in the traditional apprenticeship model requires dedicated hours to the programme to achieve proficiency. With the implementation of restricted working hours in health care systems worldwide, alternative methods of learning are required to ensure that loss of skill, and the detrimental effects this would have on patient care, is not a consequence of safer working hours. A central aspect to the benefit of simulated learning is the potential to accelerate the learning process. Factors to be evaluated should include the total time taken for development and retention of skills, stage of training that reaps the greatest benefit to simulation exposure, and whether perioperative simulation practice has any impact on clinical skills. Within the current training model, there is scope to optimize the simulation tool to ensure its use pays dividends in areas including education, technical skill obtained, and time taken.

\section{Simulated training time}

Ten studies outlined numerical metrics for VR training in the intervention group, six of which outlined total time training on VR simulation. Of these, on $\mathrm{e}^{26}$ outlined the reasoning for the time allowed. The time allocated for simulation training varied broadly, from $1 \mathrm{~h}$ total simulated training, to daily participant training for the duration of the study. Given the heterogeneity of the study designs and the tasks performed, the spectrum of time allotments allowed is expected. Previous studies have alluded to the benefit of staggered training for increased retention. ${ }^{59}$ Programmes such as the PROSPECT trial ${ }^{34}$ follow this concept with simulation training carried out in conjunction with online modules and thus staggered by design. Maertens et al. ${ }^{34}$ found this method yielded proficiency as demonstrated in the final assessment and good retention of skills at 3 months. Several studies in this trial used staggered learning methods for the VR module. ${ }^{22,26,35}$ Waterman et al. ${ }^{60}$ based training times on previously published data on improvements made per number of operations completed. Kowalewski et al. ${ }^{35}$ noted that although significant improvements were seen in the simulation group, the learning curve had not been overcome on the VR trainer. Henn et al. ${ }^{22}$ demonstrated the use of simulated arthroscopy in advancing skills, setting six sessions over 3 months to instil basic arthroscopy skills.

Gustafsson et al. ${ }^{56}$ evaluated the time taken to reach training plateaus using simulator assessment. They noted that, in addition to the time taken, the plateau level or level at which training benefit ceased to be seen, varied widely in both novices and experts and there remained a significant gap between the novices and experts. This would indicate that assessment on simulation models should be proficiency based rather than a fixed allotment of attempts or time. Eleven studies s, $21,24,25,32-34,37,39,42$ in this review used proficiency-based training. However, the results demonstrated variability in the efficacy of this model. Shore et al. ${ }^{33}$ evaluated the effect of multimodal adjacent training on clinical acumen. The authors found that although trainees improved using VR models, despite the additional simulated training in the interventional arm, they were not all able to achieve the level of competency achieved by experts; $71 \%$ of trainees reached the level of proficiency on VR by the end of the trial. Similarly, Maertens et al. ${ }^{34}$ noted not all participants exposed to VR were able to achieve the proficiency levels set by experts at the end of the trial.

\section{Stage of training}

Of the studies included in this review; six evaluated the effects of simulation on medical students ${ }^{22,23,40-42,44}$ One study looked at expert trained surgeons, ${ }^{27}$ two compared stages of training; ${ }^{20,38}$ the other studies looked at surgical trainees in a heterogeneous cohort of specialties, including ophthalmology, ${ }^{20}$ orthopaedics, ${ }^{26,39,43}$ general surger$\mathrm{y},{ }^{21,25,30,31,35 \text { gynaecology,24,33 }}$ vascular surgery, ${ }^{34} \mathrm{ENT},{ }^{37}$ and urology. ${ }^{36}$

Given the general lack of studies evaluating more than one stage of surgical experience, it is difficult to directly compare the effects of VR across levels of expertise. We have previously mentioned the learning curve that may be experienced via VR, which would indicate more impact with surgical novices. However, studies in this review demonstrated mixed findings on the impact of VR on surgical skill acquisition by medical students. Biases including interest in surgery may be potential confounders. Feudner et al. ${ }^{20}$ investigated improvement in medical students compared with surgical trainees, finding that although improvement 
was seen across the board, trainees exposed to simulation were faster to completion with no difference seen in medical students, and skill acquisition by students improved comparatively more. Lohre et al. ${ }^{38}$ compared orthopaedic trainees with experts in the use of VR in cadaveric models. Although improvement was seen in all parameters, and trainees tended to find the simulation both beneficial for development of skill and enjoyable to use, experts derived less enjoyment and ease of use of the device, with no significant agreement on the benefit of use for either trainees $(P=$ $0.10)$ or novices $(P=0.54)$. Neither group felt experts would benefit from use of the VR simulator.

\section{Impact of time on simulation on clinical acumen}

Previously published literature ${ }^{61}$ using bench-top simulation noted that, in the initial operation, a significant difference was noted between the simulation group and the control group, with loss of significance in subsequent operations, in postoperative complications and overnight stays by patients. They concluded that "warming up" may have beneficial outcomes as indicated by the finding that the conventional training group developed outcomes similar to that of the interventional arm with more exposure. Makhdom et al. ${ }^{62}$ investigated this concept by comparing the first patient's outcome with the outcomes of subsequent patients on the operating list, and noted no significant difference in outcomes between first and subsequent total hip arthroplasties on the theatre list.

Four studies looked at the use of simulated surgery and its use within a time frame of theatre. Desender et al. ${ }^{28}$ looked at the use of simulation in pre-operative planning, with trainees undergoing a simulated run through of the operation the previous evening using patient-derived metrics.

Three studies looked at the use of simulation in the immediate pre-operative period and its effect on subsequent surgical performance. ${ }^{27,29,45}$ Calatayud et al. ${ }^{29}$ evaluated the concept of warm-up benefits derived from a pre-operative VR run through. The authors found that surgical performance was improved immediately after a simulated surgery. Similarly, Moldovanu et al. ${ }^{27}$ evaluated the effect of VR on one expert surgeon's operative performance; improvement was noted with statistical significance in "tissue handling" using the global rating scale. Deuchler et al. ${ }^{45}$ noted that although performances with pre-operative simulation exposure were improved, they did not negate the variability between operations noted on a larger scale with less experienced surgeons, indicating that long-term training is required to reduce inter-operative variability in a trainee's performance. The power in these studies was generally low, and not all findings were statistically significant. Expert surgeons as well as surgical trainees were used in these study protocols and significant improvement in parameters was seen. The potential benefits of VR in fully qualified surgeons, in addition to surgical trainees, has not been thoroughly investigated; however, this finding would indicate its potential use in the consultant surgical cohort.

\section{Clinical outcomes}

Simulation training provides opportunities for surgical trainees to develop and hone skills outside theatre and in safe simulated situations, thus a decreased margin of errors would be expected in surgical practice due to the increased skillset obtained by trainees. However, to date there is a dearth of data evaluating this. One potential reason for this is that confounding factors must be taken into account when considering patient outcomes. Expert surgeons are commonly supervising surgical trainee performance intraoperatively, and their presence may have an impact on performance, and thus patient outcomes.

Of the studies included in this review, five evaluated clinical outcomes as a measure of the study. Maertens et al. ${ }^{34}$ found no statistically significant outcomes between the simulation versus online learning or versus a control group. Two studies $^{24,25}$ noted no difference in patient outcomes between the post-simulation participants and control group cohorts, with comparable complication rates and no increase in 30day mortality. Wang et al. ${ }^{36}$ evaluated the rates of complications found in laparoscopic varicocelectomies in the calculation of learning curves. The use of VR was found to have similar complications rates as box-training methods. No control was used for comparison; a limitation to the study.

Desender et al. ${ }^{28}$ evaluated the effects of using patient specifications within a simulated environment in the pre-operative period. They found a significant difference in the occurrence of minor and major errors in the intra-operative phase, with no difference in 30-day mortality between the interventional arm and the control group. Further research regarding the impact of simulation training on patient outcomes is warranted.

\section{Limitations}

Broad heterogeneity existed in methods and participant characteristics in studies included in this review. The type of simulator used, the method of analysis of participants, and the procedures involved are diverse enough to make direct comparisons difficult. Although similar assessment tools were used in the studies, the different levels of experience of participants evaluated reduce the comparability of these validated tools. Only one author was involved in study inclusion, therefore the selection process was subject to bias. 


\section{Conclusion}

Use of VR training has become an accepted form of adjunctive surgical training. Results from this review indicate that, despite mixed findings in its predictive value compared with a sole learning tool, well-powered studies in which VR was used in surgical curricula in conjunction with other forms of surgical education yielded positive results, with predictive validity demonstrated in participants. The effects of VR on patient outcomes have yet to be established. Interestingly, the use of VR in improving non-technical skills, with particular focus on the benefits of immersive training over traditional forms of education, were demonstrated in this review. The use of VR in the pre-operative setting and its use in creating immersive operative simulations for trainees warrants further research.

\section{Conflicts of interest}

None declared.

\section{References}

1. Hanks JB. Simulation in surgical education: influences of and opportunities for the Southern Surgical Association. J Am Coll Surg 2019; 228(4): 317-328. https://doi.org/10.1016/j.jamcollsurg.2018.12.029.

2. Agha RA, Fowler AJ. The role and validity of surgical simulation. Int Surg 2015; 100(2): 350-357. https://doi.org/10. 9738/INTSURG-D-14-00004.1.

3. Robertson V, Davies R. Provision of simulation-based training (SBT) within UK vascular surgery training programmes. Surgeon 2019; 17(6): 321-325. https://doi.org/10.1016/j.surge. 2018.10.001.

4. Hurreiz $\mathrm{H}$. The evolution of surgical training in the UK. Adv Med Educ Pract 2019; 10: 163-168. https://doi.org/10. 2147/AMEP.S189298.

5. Seymour NE, Gallagher AG, Roman SA, O'Brien MK, Bansal VK, Andersen DK, et al. Virtual reality training improves operating room performance: results of a randomized, double-blinded study. Ann Surg 2002; 236(4): 458-64. https://doi.org/10.1097/00000658-200210000-00008.

6. Vera AM, Russo M, Mohsin A, Tsuda S. Augmented reality telementoring (ART) platform: a randomized controlled trial to assess the efficacy of a new surgical education technology. Surg Endosc 2014; 28(12): 3467-3472. https://doi.org/10. 1007/s00464-014-3625-4.

7. Kogan M, Klein SE, Hannon CP, Nolte MT. Orthopaedic education during the COVID-19 pandemic. J Am Acad Orthop Surg 2020; 28(11): e456-e64. https://doi.org/10. 5435/JAAOS-D-20-00292.
8. Agyeman KD, Summers SH, Massel DH, Mouhanna J, Aiyer A, Dodds SD. Innovation in orthopaedic surgery education: novel tools for modern times. J Am Acad Orthop Surg 2020; 28(18): e782-e792 https://doi.org/10.5435/JAAOS-D-19-00411.

9. Gupta N, Agrawal H. COVID 19 and surgical education: time for innovations. Indian J Surg 2020: 1-2. https://doi.org/10. 1007/s12262-020-02422-5.

10. Rothschild P, Richardson A, Franzco JB, Franzco RC. Does virtual reality simulation training result in fewer real-life cataract surgery complications? A systematic literature review. J Cataract Refract Surg 2020. https://doi.org/10.1097/j.jcrs. 0000000000000323.

11. Lamblin G, Thiberville G, Druette L, Moret S, Couraud S, Martin X, et al. Virtual reality simulation to enhance laparoscopic salpingectomy skills. J Gynecol Obstet Hum Reprod 2020; 49(3): 101685. https://doi.org/10.1016/j.jogoh.2020. 101685.

12. Fiani B, De Stefano F, Kondilis A, Covarrubias C, Reier L, Sarhadi K. Virtual reality in neurosurgery: "can you see it?"-a review of the current applications and future potential. World Neurosurg 2020; 141: 291-298. https://doi.org/10.1016/j.wneu. 2020.06.066.

13. Hooper J, Tsiridis E, Feng JE, Schwarzkopf R, Waren D, Long WJ, et al. Virtual reality simulation facilitates resident training in total hip arthroplasty: a randomized controlled trial. J Arthroplasty 2019; 34(10): 2278-2283. https://doi.org/10. 1016/j.arth.2019.04.002.

14. Walsh CM, Sherlock ME, Ling SC, Carnahan H. Virtual reality simulation training for health professions trainees in gastrointestinal endoscopy. Cochrane Database Syst Rev 2012; (6): CD008237. https://doi.org/10.1002/14651858.CD008237. pub2.

15. Rudarakanchana N, Van Herzeele I, Desender L, Cheshire NJ. Virtual reality simulation for the optimization of endovascular procedures: current perspectives. Vasc Health Risk Manag 2015; 11: 195-202. https://doi.org/10.2147/VHRM.S46194.

16. Meling TR. The impact of surgical simulation on patient outcomes: a systematic review and meta-analysis. Neurosurg Rev 2020. https://doi.org/10.1007/s10143-020-01314-2.

17. Alaker M, Wynn GR, Arulampalam T. Virtual reality training in laparoscopic surgery: a systematic review \& meta-analysis. Int J Surg 2016; 29: 85-94. https://doi.org/10.1016/j.ijsu.2016. 03.034 .

18. Sterne JAC, Savović J, Page MJ, Elbers RG, Blencowe NS, Boutron I, et al. RoB 2: a revised tool for assessing risk of bias in randomised trials. BMJ 2019; 366: 14898. https://doi. org/10.1136/bmj.14898.

19. Sterne JA, Hernán MA, Reeves BC, Savović J, Berkman ND, Viswanathan M, et al. ROBINS-I: a tool for assessing risk of bias in non-randomised studies of interventions. BMJ 2016; 355: i4919. https://doi.org/10.1136/bmj.i4919. 
20. Feudner EM, Engel C, Neuhann IM, Petermeier K, BartzSchmidt KU, Szurman P. Virtual reality training improves wet-lab performance of capsulorhexis: results of a randomized, controlled study. Graefes Arch Clin Exp Ophthalmol 2009; 247(7): 955-963. https://doi.org/10.1007/s00417-008-1029-7.

21. Martin JR, Stefanidis D, Dorin RP, Goh AC, Satava RM, Levy JS. Demonstrating the effectiveness of the fundamentals of robotic surgery (FRS) curriculum on the RobotiX Mentor Virtual Reality Simulation Platform. J Robot Surg 2021; 15(2): 187-193. https://doi.org/10.1007/s11701-020-01085-4.

22. Henn RF, Shah N, Warner JJ, Gomoll AH. Shoulder arthroscopy simulator training improves shoulder arthroscopy performance in a cadaveric model. Arthroscopy 2013; 29(6): 982985. https://doi.org/10.1016/j.arthro.2013.02.013.

23. Wang KC, Bernardoni ED, Cotter EJ, Cole BJ, Verma NN, Romeo AA, et al. Impact of simulation training on diagnostic arthroscopy performance: a randomized controlled trial. Arthrosc Sports Med Rehabil 2019; 1(1): e47-e57. https://doi.org/10.1016/j.asmr.2019.07.002.

24. Ahlborg L, Hedman L, Nisell H, Felländer-Tsai L, Enochsson L. Simulator training and non-technical factors improve laparoscopic performance among OBGYN trainees. Acta Obstet Gynecol Scand 2013; 92(10): 1194-1201. https://doi. org/10.1111/aogs.12218.

25. Beyer-Berjot L, Pucher P, Patel V, Hashimoto DA, Ziprin P, Berdah $\mathrm{S}$, et al. Colorectal surgery and enhanced recovery: impact of a simulation-based care pathway training curriculum. J Visc Surg 2017; 154(5): 313-320. https://doi.org/10. 1016/j.jviscsurg.2017.02.003.

26. Waterman BR, Martin KD, Cameron KL, Owens BD, Belmont PJ. Simulation training improves surgical proficiency and safety during diagnostic shoulder arthroscopy performed by residents. Orthopedics 2016; 39(3): e479-e485. https://doi. org/10.3928/01477447-20160427-02.

27. Moldovanu R, Târcoveanu E, Dimofte G, Lupaşcu C, Bradea C. Preoperative warm-up using a virtual reality simulator. JSLS 2011; 15(4): 533-538. https://doi.org/10.4293/1086808 $11 X 13176785204409$.

28. Deuchler S, Wagner C, Singh P, Müller M, Al-Dwairi R, Benjilali R, et al. Clinical efficacy of simulated vitreoretinal surgery to prepare surgeons for the upcoming intervention in the operating room. PLoS One 2016; 11(3): e0150690. https://doi.org/10.1371/journal.pone.0150690.

29. Desender LM, Van Herzeele I, Lachat ML, Rancic Z, Duchateau J, Rudarakanchana N, et al. Patient-specific rehearsal before EVAR: influence on technical and nontechnical operative performance. A randomized controlled trial. Ann Surg 2016; 264(5): 703-709. https://doi.org/10.1097/SLA. 0000000000001871.

30. Calatayud D, Arora S, Aggarwal R, Kruglikova I, Schulze S, Funch-Jensen $\mathrm{P}$, et al. Warm-up in a virtual reality environment improves performance in the operating room. Ann Surg
2010; 251(6): 1181-1185. https://doi.org/10.1097/SLA. 0b013e3181deb630.

31. Palter VN, Grantcharov TP. Development and validation of a comprehensive curriculum to teach an advanced minimally invasive procedure: a randomized controlled trial. Ann Surg 2012; 256(1): 25-32. https://doi.org/10.1097/SLA.0b013e318258f5aa.

32. Palter VN, Orzech N, Reznick RK, Grantcharov TP. Validation of a structured training and assessment curriculum for technical skill acquisition in minimally invasive surgery: a randomized controlled trial. Ann Surg 2013; 257(2): 224-230. https://doi.org/10.1097/SLA.0b013e31827051cd.

33. Satava RM, Stefanidis D, Levy JS, Smith R, Martin JR, Monfared S, et al. Proving the effectiveness of the fundamentals of robotic surgery (FRS) skills curriculum: a singleblinded, multispecialty, multi-institutional randomized control trial. Ann Surg 2020; 272(2): 384-392. https://doi.org/10. 1097/SLA.0000000000003220.

34. Shore EM, Grantcharov TP, Husslein H, Shirreff L, Dedy NJ, McDermott CD, et al. Validating a standardized laparoscopy curriculum for gynecology residents: a randomized controlled trial. Am J Obstet Gynecol 2016; 215(2): 204.e1-e11. https://doi.org/10.1016/j.ajog.2016.04.037.

35. Maertens H, Aggarwal R, Moreels N, Vermassen F, Van Herzeele I. A proficiency based stepwise endovascular curricular training (PROSPECT) program enhances operative performance in real life: a randomised controlled trial. Eur J Vasc Endovasc Surg 2017; 54(3): 387-396. https://doi.org/10.1016/j. ejvs.2017.06.011.

36. Kowalewski KF, Garrow CR, Proctor T, Preukschas AA, Friedrich M, Müller PC, et al. LapTrain: multi-modality training curriculum for laparoscopic cholecystectomy-results of a randomized controlled trial. Surg Endosc 2018; 32(9): 38303838. https://doi.org/10.1007/s00464-018-6110-7.

37. Wang Z, Ni Y, Zhang Y, Jin X, Xia Q, Wang H. Laparoscopic varicocelectomy: virtual reality training and learning curve. JSLS 2014; 18(3): e2014.00258. https://doi.org/10.4293/JSLS. 2014.00258.

38. Fried MP, Sadoughi B, Gibber MJ, Jacobs JB, Lebowitz RA, Ross DA, et al. From virtual reality to the operating room: the endoscopic sinus surgery simulator experiment. Otolaryngol Head Neck Surg 2010; 142(2): 202-207. https://doi.org/10. 1016/j.otohns.2009.11.023.

39. Lohre R, Bois AJ, Athwal GS, Goel DP, Canadian Shoulder and Elbow Society (CSES). Improved complex skill acquisition by immersive virtual reality training: a randomized controlled trial. J Bone Joint Surg Am 2020; 102(6): e26. https://doi. org/10.2106/JBJS.19.00982.

40. Cannon WD, Garrett WE, Hunter RE, Sweeney HJ, Eckhoff DG, Nicandri GT, et al. Improving residency training in arthroscopic knee surgery with use of a virtual-reality simulator. A randomized blinded study. J Bone Joint Surg Am 2014; 96(21): 1798-1806. https://doi.org/10.2106/JBJS.N.00058. 
41. Nemani A, Ahn W, Cooper C, Schwaitzberg S, De S. Convergent validation and transfer of learning studies of a virtual reality-based pattern cutting simulator. Surg Endosc 2018; 32(3): 1265-1272. https://doi.org/10.1007/s00464-017-5802-8.

42. Nickel F, Brzoska JA, Gondan M, Rangnick HM, Chu J, Kenngott $\mathrm{HG}$, et al. Virtual reality training versus blended learning of laparoscopic cholecystectomy: a randomized controlled trial with laparoscopic novices. Medicine (Baltimore) 2015; 94(20): e764. https://doi.org/10.1097/MD.0000000000 000764.

43. Van Bruwaene S, Schijven MP, Napolitano D, De Win G, Miserez M. Porcine cadaver organ or virtual-reality simulation training for laparoscopic cholecystectomy: a randomized, controlled trial. J Surg Educ 2015; 72(3): 483-490. https://doi. org/10.1016/j.jsurg.2014.11.015.

44. Rebolledo BJ, Hammann-Scala J, Leali A, Ranawat AS. Arthroscopy skills development with a surgical simulator: a comparative study in orthopaedic surgery residents. Am J Sports Med 2015; 43(6): 1526-1529. https://doi.org/10. $1177 / 0363546515574064$.

45. Banaszek D, You D, Chang J, Pickell M, Hesse D, Hopman WM, et al. Virtual reality compared with bench-top simulation in the acquisition of arthroscopic skill: a randomized controlled trial. J Bone Joint Surg Am 2017; 99(7): e34. https://doi.org/10.2106/JBJS.16.00324.

46. Gasperin BDM, Zanirati T, Cavazzola LT. Can virtual reality be as good as operating room training? Experience from a residency program in general surgery. Arq Bras Cir Dig 2018; 31(4): e1397. https://doi.org/10.1590/0102-67202018 $0001 \mathrm{e} 1397$.

47. Sugand K, Akhtar K, Khatri C, Cobb J, Gupte C. Training effect of a virtual reality haptics-enabled dynamic hip screw simulator. Acta Orthop 2015; 86(6): 695-701. https://doi. org/10.3109/17453674.2015.1071111.

48. Rahm S, Wieser K, Bauer DE, Waibel FW, Meyer DC, Gerber $\mathrm{C}$, et al. Efficacy of standardized training on a virtual reality simulator to advance knee and shoulder arthroscopic motor skills. BMC Musculoskelet Disord 2018; 19(1): 150. https://doi. org/10.1186/s12891-018-2072-0.

49. Hoopes S, Pham T, Lindo FM, Antosh DD. Home surgical skill training resources for obstetrics and gynecology trainees during a pandemic. Obstet Gynecol 2020; 136(1): 56-64. https://doi.org/10.1097/AOG.0000000000003931.

50. Lam CK, Sundaraj K, Sulaiman MN, Qamarruddin FA. Virtual phacoemulsification surgical simulation using visual guidance and performance parameters as a feasible proficiency assessment tool. BMC Ophthalmol 2016; 16: 88. https://doi. org/10.1186/s12886-016-0269-2.

51. Pedowitz RA, Esch J, Snyder S. Evaluation of a virtual reality simulator for arthroscopy skills development. Arthroscopy 2002; 18(6): E29. https://doi.org/10.1053/jars.2002.33791.
52. Mahmood T, Scaffidi MA, Khan R, Grover SC. Virtual reality simulation in endoscopy training: current evidence and future directions. World J Gastroenterol 2018; 24(48): 5439-5445. https://doi.org/10.3748/wjg.v24.i48.5439.

53. Khan R, Plahouras J, Johnston BC, Scaffidi MA, Grover SC, Walsh CM. Virtual reality simulation training for health professions trainees in gastrointestinal endoscopy. Cochrane Database Syst Rev 2018; 8: CD008237. https://doi.org/10. 1002/14651858.CD008237.pub3.

54. Redondo ML, Christian DR, Gowd AK, Cabarcas BC, Cvetanovich G, Bach BR, et al. The effect of triangulation simulator training on arthroscopy skills: a prospective randomized controlled trial. Arthrosc Sports Med Rehabil 2020; 2(2): e59-e70. https://doi.org/10.1016/j.asmr.2019.10.010.

55. Pusic M, Pecaric M, Boutis K. How much practice is enough? Using learning curves to assess the deliberate practice of radiograph interpretation. Acad Med 2011; 86(6): 731-736. https://doi.org/10.1097/ACM.0b013e3182178c3c.

56. Gustafsson A, Pedersen P, Rømer TB, Viberg B, Palm H, Konge L. Hip-fracture osteosynthesis training: exploring learning curves and setting proficiency standards. Acta Orthop 2019; 90(4): 348-353. https://doi.org/10.1080/17453674.2019. 1607111.

57. Duarte RJ, Cury J, Oliveira LC, Srougi M. Establishing the minimal number of virtual reality simulator training sessions necessary to develop basic laparoscopic skills competence: evaluation of the learning curve. Int Braz J Urol 2013; 39(5): 712719. https://doi.org/10.1590/S1677-5538.IBJU.2013.05.14.

58. Dedy NJ, Zevin B, Bonrath EM, Grantcharov TP. Current concepts of team training in surgical residency: a survey of North American program directors. J Surg Educ 2013; 70(5): 578-584. https://doi.org/10.1016/j.jsurg.2013.04.011.

59. Moulton CA, Dubrowski A, Macrae H, Graham B, Grober E, Reznick R. Teaching surgical skills: what kind of practice makes perfect? a randomized, controlled trial. Ann Surg 2006; 244(3): 400-409. https://doi.org/10.1097/01.sla. 0000234808.85789.6a.

60. Martin KD, Cameron K, Belmont PJ, Schoenfeld A, Owens BD. Shoulder arthroscopy simulator performance correlates with resident and shoulder arthroscopy experience. J Bone Joint Surg Am 2012; 94(21): e160. https://doi.org/10. 2106/JBJS.L.00072.

61. Zendejas B, Cook DA, Bingener J, Huebner M, Dunn WF, Sarr MG, et al. Simulation-based mastery learning improves patient outcomes in laparoscopic inguinal hernia repair: a randomized controlled trial. Ann Surg 2011; 254(3): 502511. https://doi.org/10.1097/SLA.0b013e31822c6994.

62. Makhdom AM, Almaawi A, Tanzer D, Tanzer M. Does warming up improve surgical outcome in total hip arthroplasty? Eur J Orthop Surg Traumatol 2015; 25(8): 1265-1269. https://doi. org/10.1007/s00590-015-1679-1. 